\title{
The incidence and risk factors of venous thromboembolism in Japanese inpatients with inflammatory bowel disease: a retrospective cohort study
}

\author{
Katsuyoshi Ando $^{1 *}$, Mikihiro Fujiya $^{1 *}$, Yoshiki Nomura ${ }^{1}$, Yuhei Inaba ${ }^{2}$, Yuuya Sugiyama ${ }^{1}$, Takuya Iwama ${ }^{1}$, \\ Masami Ijiri ${ }^{1}$, Keitaro Takahashi ${ }^{1}$, Kazuyuki Tanaka ${ }^{1}$, Aki Sakatani ${ }^{1}$, Nobuhiro Ueno ${ }^{1}$, Shin Kashima ${ }^{1}$, \\ Kentaro Moriichi ${ }^{1}$, Yusuke Mizukami ${ }^{1}$, Toshikatsu Okumura ${ }^{1}$ \\ ${ }^{1}$ Division of Gastroenterology and Hematology/Oncology, Department of Medicine, Asahikawa Medical University, Asahikawa, ${ }^{2}$ Department \\ of Gastroenterology, Asahikawa City Hospital, Asahikawa, Japan
}

Background/Aims: Venous thromboembolism (VTE) is a major extraintestinal manifestation in inflammatory bowel disease (IBD), regarded as an independent risk factor for VTE according to reports from Western countries. However, the incidence and risk factors of VTE in Asian IBD patients are not fully understood. We aimed to reveal the incidence and risk factors of VTE in Japanese IBD inpatients. Methods: The incidence of VTE in inpatients with IBD ( $\mathrm{n}=340)$, gastrointestinal cancers ( $\mathrm{n}=557)$, and other gastrointestinal diseases $(n=569)$ treated at our hospital from 2009 to 2013 was retrospectively investigated. The characteristics and laboratory data of IBD inpatients with and without VTE were compared in univariate and multivariate analyses. Clinical courses of VTE in IBD were surveyed. Results: VTE was detected in 7.1\% of IBD inpatients, significantly higher than in gastrointestinal cancer inpatients $(2.5 \%)$ and inpatients with other gastrointestinal diseases $(0.88 \%)$. The incidence of VTE in ulcerative colitis (UC) patients (16.7\%) was much higher than that in those with Crohn's disease (3.6\%). In the univariate analysis, the risk factors were an older age, central venous catheter, prednisolone, surgery, low serum albumin, high serum C-reactive protein and D-dimer. According to a multivariate analysis, $>50$ years of age and surgery were the only risk factors. The in-hospital mortality rate of IBD inpatients with VTE was $4.2 \%$. Conclusions: The incidence of VTE with IBD, especially UC, was found to be high compared with other digestive disease, which was almost equivalent to that of Western countries. The efficacy of prophylaxis needs to be investigated in Asian IBD patients. (Intest Res 2018;16:416-425)

Key Words: Venous thromboembolism; Inflammatory bowel disease; Risk factors

\section{INTRODUCTION}

Inflammatory bowel disease (IBD), including UC and CD, is refractory intestinal inflammation, the etiology of which is unclear. Various procoagulant states in IBD populations have been reported, including the activation and aggregation of

Received October 7, 2017. Revised January 2, 2018.

Accepted January 3, 2018. Published online July 11, 2018

Correspondence to Mikihiro Fujiya, Division of Gastroenterology and

Hematology/Oncology, Department of Medicine, Asahikawa Medical

University, 2-1 Midorigaoka-higashi, Asahikawa 078-8510, Japan. Tel: +81-

166-68-2462, Fax: +81-166-68-2469, E-mail: fjym@asahikawa-med.ac.jp

*These authors contributed equally to this study. platelets, increased procoagulant factors and decreased anticoagulant factors. ${ }^{1-3}$ Clinical investigations have shown that venous thromboembolism (VTE) is a major extraintestinal manifestation with IBD in Western populations, with an incidence in IBD patients of $1.0 \%$ to $7.7 \%{ }^{4-7}$ Recent large-scale cohort studies in Western countries have reported the incidence rate of VTE in IBD patients to be twice to triple that in healthy subjects. ${ }^{1}$ Miehsler et al. ${ }^{8}$ showed that the risk of VTE is 3.6-fold higher than in age- and sex-matched patients with other systemic inflammatory diseases (rheumatoid arthritis) and intestinal inflammation (celiac disease). Therefore, IBD is regarded as an independent risk factor for VTE.

\footnotetext{
๑ Copyright 2018. Korean Association for the Study of Intestinal Diseases. All rights reserved.

This is an Open Access article distributed under the terms of the Creative Commons Attribution Non-Commercial License (http://creativecommons.org/licenses/by-nc/4.0)

which permits unrestricted non-commercial use, distribution, and reproduction in any medium, provided the original work is properly cited.
} 
Hospitalization, a diagnosis of UC, fistulizing type CD, $\mathrm{CD}$ with colonic involvement, older age, flare and bowel resection have been shown to be risk factors of VTE in IBD patients in Western populations. ${ }^{2,9,10}$ VTE is regarded to cause organ failure and death in IBD patients, particularly those with portal vein thrombosis (PVT), cerebral vein sinus thrombosis, and pulmonary thromboembolism. ${ }^{11,12}$ Nguyen and $\mathrm{Sam}^{9}$ reported that VTE in inpatients with IBD occurred at younger ages and had a higher mortality than in patients without IBD. Therefore, elucidating the incidence and risk factors of IBD-associated VTE is essential for the prevention of VTE in patients with IBD. Generally, the incidence of VTE is thought to be lower in Asia than in Western countries because of the lower BMI and lower incidences of VTE risk factors, such as hyperlipidemia, among Asians. Only one single arm prospective study with a small number of patients has been reported in an Asian population. ${ }^{13}$ It is urgent to clarify the incidence and risk factors of VTE, as well as to establish therapeutic and preventive strategies for VTE in Asian IBD patients.

The present retrospective cohort study was proposed to

Table 1. Characteristics of IBD Inpatients

\begin{tabular}{lccc}
\hline \multicolumn{1}{c}{ Characteristics } & $\begin{array}{c}\text { IBD } \\
(\mathbf{n}=\mathbf{3 4 0})\end{array}$ & $\begin{array}{c}\text { UC } \\
(\mathbf{n}=\mathbf{8 9})\end{array}$ & $\begin{array}{c}\text { CD } \\
(\mathbf{n}=\mathbf{2 5 1})\end{array}$ \\
\hline Age (yr) & $39.4 \pm 15.4$ & $40.5 \pm 20.4$ & $36.2 \pm 12.0$ \\
Sex (male/female) & $234 / 105$ & $50 / 39$ & $184 / 67$ \\
BMl (kg/m²) & $20.4 \pm 3.05$ & $20.6 \pm 3.5$ & $19.8 \pm 2.7$ \\
Disease duration (yr) & $11.6 \pm 9.0$ & $5.5 \pm 6.5$ & $13.7 \pm 8.8$ \\
Disease type & - & $0 / 10 / 79^{\mathrm{a}}$ & $92 / 151 / 8^{\mathrm{b}}$ \\
Disease activity & - & $8.4 \pm 3.9^{\mathrm{c}}$ & $180.2 \pm 78.2^{\mathrm{d}}$ \\
Smoking & $88(25.8)$ & $7(7.9)$ & $81(32.3)$ \\
Past history of thrombosis & $11(3.2)$ & $2(2.2)$ & $9(3.6)$ \\
CV catheter & $97(28.5)$ & $28(31.4)$ & $69(27.5)$ \\
Predonisolone & $40(11.8)$ & $33(37.1)$ & $7(2.7)$ \\
\hline Tacrolimus/cyclosporine & $12(3.5)$ & $12(13.5)$ & 0 \\
Thiopurine & $105(30.9)$ & $21(23.6)$ & $84(33.5)$ \\
Anti-TNF- $\alpha$ antibody & $227(66.8)$ & $28(31.4)$ & $199(79.3)$ \\
Plasmacytoapheresis & $14(4.1)$ & $12(13.5)$ & $2(0.8)$ \\
Surgery & $51(15.0)$ & $8(9.0)$ & $43(17.1)$ \\
\hline
\end{tabular}

Values are presented as mean \pm SD or number (\%).

${ }^{a}$ Proctitis/left sided colitis/total colitis type.

${ }^{\natural}$ Ileitis/ileo-colitis/colitis type.

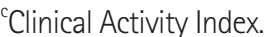

${ }^{\mathrm{d}} \mathrm{CDAl}$.

$\mathrm{CV}$, central venous catheters; TNF, tumor necrosis factor. reveal the incidence and risk factors of IBD-associated VTE compared with that in gastrointestinal (GI) cancer and other digestive diseases in a Japanese population.

\section{METHODS}

\section{Study Population}

A total of 1,466 patients with GI disease admitted to Asahikawa Medical University Hospital from January 2009 to December 2013 and who underwent contrast-enhanced CT (CECT) and/or ultrasonography (US) at least once were enrolled. Of these, 340 patients with IBD (89 with UC, 251 with CD), 557 patients with GI cancer (esophageal cancer, gastric cancer, colon cancer and small intestinal cancer), and 569 patients with other GI disorders (e.g., ileus, GI tract bleeding, infectious colitis, ischemic colitis and colon adenoma) were ultimately included in the study. In cases of multiple admissions, the same patients were regarded as different individuals if the duration of admission exceeded 1 month. The actual number of patients enrolled in this study was 183 patients with IBD (68 with UC and 115 with CD; number of admissions, 1-11), 365 with GI cancer (number of admissions, 1-16) and 464 with other GI disorders (number of admissions, 1-5). Patient characteristics and laboratory data on admission among inpatients among IBD (Tables 1 and 2), GI cancer and other GI disorders (Table 2) enrolled in this study were sufficiently described in their clinical records. The study was approved by the Ethics Comittee of Asahikawa Medical Unversity (IRB No. 17092) and performed in accordance with the principles of the Declaration of Helsinki. The informed consent was waived.

Table 2. Characteristics of Inpatients with IBD, GI Cancer and Other GI Diseases

\begin{tabular}{lccc}
\hline \multicolumn{1}{c}{ Characteristics } & $\begin{array}{c}\text { IBD } \\
(\mathbf{n = 3 4 0 )}\end{array}$ & $\begin{array}{c}\text { GI cancer } \\
(\mathbf{n}=\mathbf{5 5 7})\end{array}$ & $\begin{array}{c}\text { Other GI } \\
\text { diseases } \\
(\mathbf{n}=\mathbf{5 6 9})\end{array}$ \\
\hline Age (yr) & $39.4 \pm 15.4$ & $69.9 \pm 10.8$ & $60.3 \pm 18.8$ \\
Sex (male/female) & $234 / 105$ & $368 / 189$ & $330 / 239$ \\
BMI $\left(\mathrm{kg} / \mathrm{m}^{2}\right)$ & $20.4 \pm 3.05$ & $22.9 \pm 17.0$ & $22.3 \pm 4.0$ \\
History of thrombosis & $11(3.2)$ & $6(1.1)$ & $11(1.9)$ \\
Usage of antithrombotic drugs & $12(3.5)$ & $60(10.8)$ & $70(12.3)$ \\
CV catheter & $97(28.5)$ & $172(30.9)$ & $86(15.1)$ \\
Surgery & $51(15.0)$ & $63(11.3)$ & $50(8.8)$ \\
\hline
\end{tabular}

Values are presented as mean \pm SD or number (\%).

$\mathrm{Gl}$, gastrointestinal; $\mathrm{CV}$, central venous. 


\section{Surveillance and Prophylaxis of VTE}

The presence or absence of VTE was surveyed by CECT and/or US. In all cases scanned by CECT, the scanned area stretched from the chest to the pelvis, the level of which was the pulmonary artery to the proximal iliac vein. The scanned area was extended to the lower limbs and soles in suspicious cases that needed to be screened for VTE. In all cases scanned by US, the scanned area stretched from the superior vena cava to the venous in lower limbs. CECT scans from the chest to the lower limbs and soles were added mainly for the surveillance of VTE sites other than the lower limbs in all cases of VTE detected by US. VTE was diagnosed as positive if either CECT or US findings were positive for thrombus despite discrepancies in the presence or absence of thrombi between those 2 procedures. VTE was diagnosed when CECT showed defects in the venous or around the central venous catheter, regardless of the complaint of the patients. CECT or US was performed when VTE was suspected due to symptoms such as swelling, redness and pain of upper or lower limbs, respiratory discomfort, headache (indicated cerebral venous sinus thrombosis), and poor dropping of the CV catheter. Cases with VTE detected in above situations were defined as symptomatic VTE in this study. In addition, asymptomatic cases of VTE that were incidentally detected by CECT and/or US performed for the diagnosis and evaluation of a disease on and/or after admission was included in this study. The incidence rate and sites of VTE were investigated. The CT findings of cases who developed VTE at various sites are shown in Fig. 1. Patient characteristics and laboratory data of blood examinations in the 2 groups of IBD with and without VTE were compared to determine the risk factors for univariate and multivariate analyses. In addition, treatment against VTE, hemorrhagic complications associated with anticoagulant therapy, and mortality related to VTE were surveyed in IBD patients with VTE. Prophylaxis for VTE was performed as described in the manual for VTE developed at our hospital. Prophylaxis was carried out for patients with surgical treatment for IBD, cancer or other disorders using mechanical or pharmacological methods. However, in patients admitted to the department of internal medicine, no pharmacological prophylaxis was routinely performed because of the lack of adequate guidance in our country. However, mechanical prophylaxis was
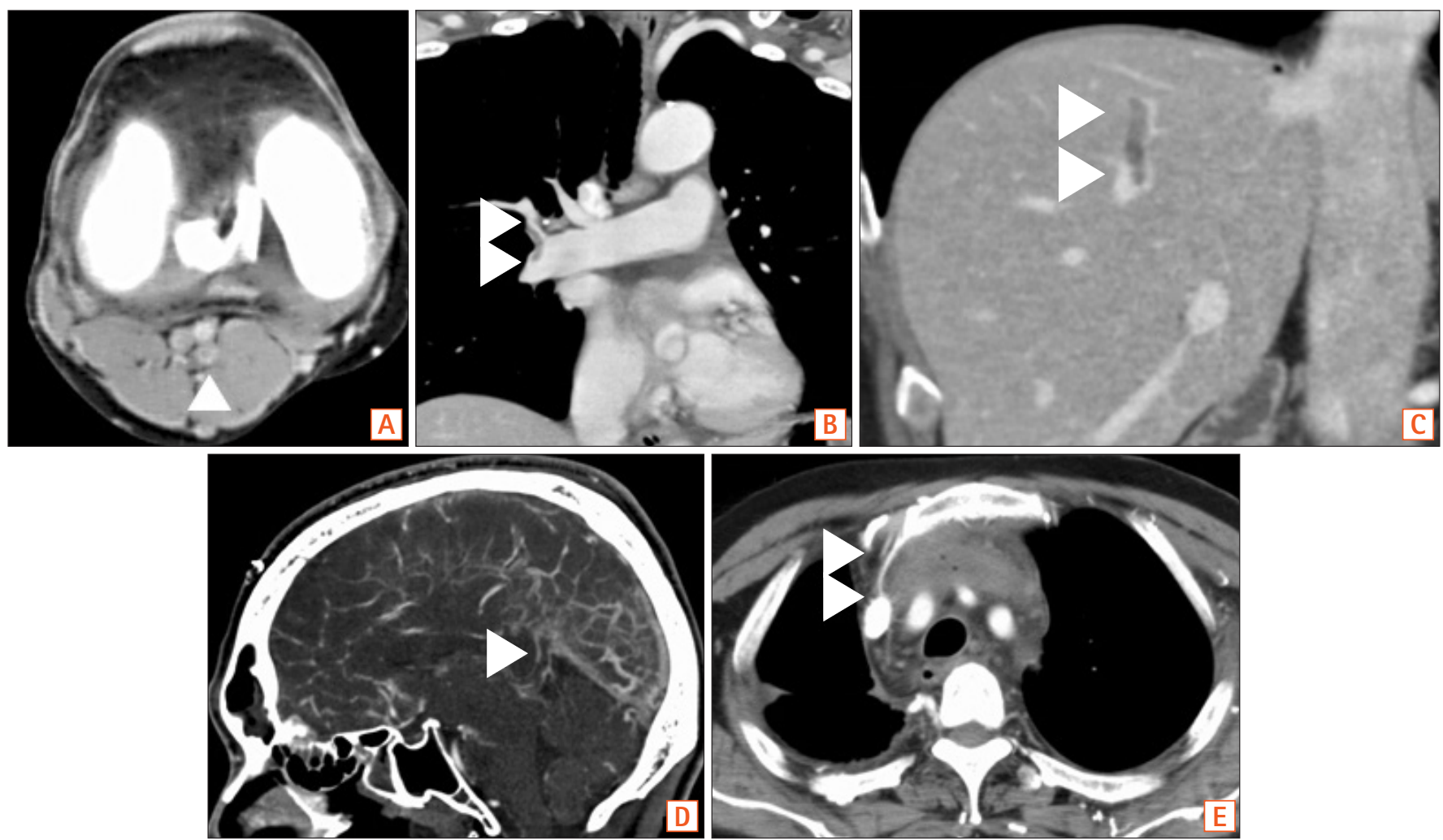

Fig. 1. The CT findings of cases with venous thromboembolism (VTE) developing in various site. In our study, VTE was developing in various site, which included venous in lower limbs (A), pulmonary artery (B), portal and mesenteric venous (C), cerebral venous sinus (D) and catheter indwelling site (E). Thrombosis was shown with arrowheads in each image. 
carried out for patients judged by their attending physicians as being adaptive. In patients with a history of VTE, the differential diagnosis between currently developing thrombus and previously developed thrombus was achieved based on the findings of CECT and US, which consisted of the CT value, presence or absence of organization, mural defects and bands of veins on CECT and the brightness, presence or absence of floating, shape and blood flow of veins on US. In patients receiving anticoagulant therapy, the decrease or

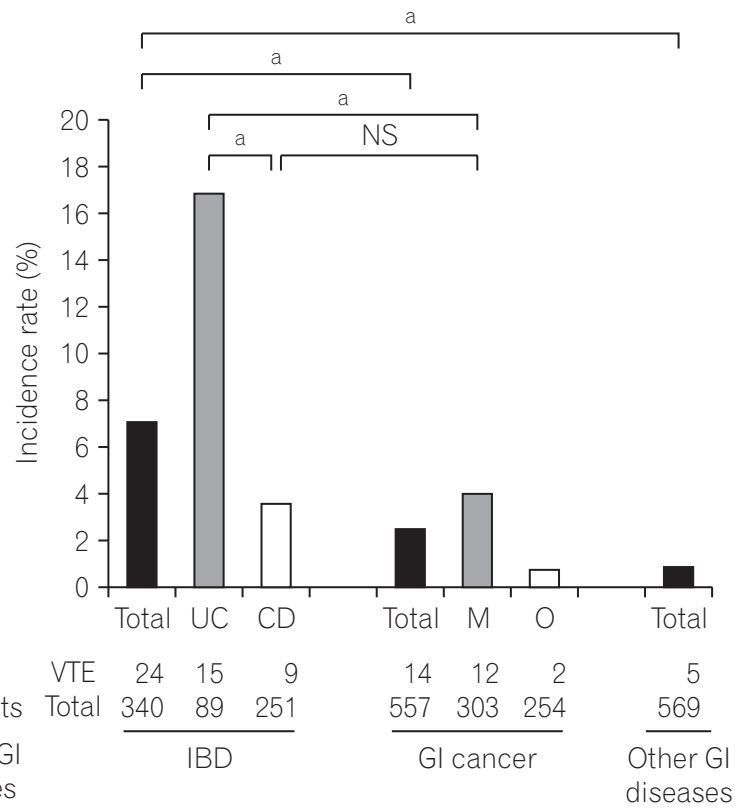

Fig. 2. Incidence of venous thromboembolism (VTE) in inpatients. The incidence of VTE in IBD inpatients was 7.1\% (24/340), which was significantly higher than in gastrointestinal (GI) cancer and other $\mathrm{Gl}$ disease inpatients $\left(P<0.0001^{\text {a }}\right.$, respectively). The incidence of VTE in UC $(16.9 \%, 15 / 89)$ was significantly higher than that in CD $(3.6 \%, 9 / 251)$ $\left(P<0.0001^{\mathrm{a}}\right)$. The rate of VTE in UC was considerably higher than that in cancer with distant metastasis or chemotherapy, although that in $\mathrm{CD}$ was almost equivalent to that in cancer with distant metastasis or chemotherapy in our study. M, cancer with distant metastasis or chemotherapy; 0 , others. disappearance of the VTE was confirmed after the therapy. In patients with a history of indwelling venous catheter, a thrombus sticking around the catheter was regarded as catheter-related "current" thrombus when CECT or US was performed with an indwelling venous catheter. In cases of VTE detected after the removal of the catheter, "current" thrombus at the site of the previously indwelling catheter was regarded as catheter-related thrombus.

\section{Patient Characteristics and Laboratory Data}

Patient characteristics and laboratory data were collected in IBD with or without VTE from medical records. The characteristics were age, sex, BMI, disease duration, type of disease extent (UC: total colitis, left-sided, and proctitis type; CD: ileitis, ileo-colitis, and colitis type), disease activity on admission (UC: Clinical Activity Index; CD: CDAI), smoking, history of thrombosis, presence or absence of central venous alimentation, type of medical therapy treated in admission (prednisolone, immunomodulators, anti-tumor necrosis factor- $\alpha$ antibody, cytapheresis), presence or absence of abdominal surgery (within 2 weeks before or after surgery, consisting of total colectomy or partial resection of small intestine or colon), and systemic complications (diabetes mellitus, dyslipidemia, hypertension). Laboratory data on admission were the numbers of white blood cells and platelets, hemoglobin, serum levels of CRP, total protein, albumin, BUN, total cholesterol, triglyceride, hemoglobin Alc, ESR (1 hour), and, fibrinogen, D-dimer, fibrin degenerative product, anti-thrombin III, INR of PT, and active partial thromboplastin time.

\section{Treatment for VTE}

In accordance with the guidelines for treating VTE, patients with VTE were referred to specialists and treated with anticoagulant, fibrinolytic therapy, indwelling of inferior

Table 3. Incidence Site of Venous Thrombosis in IBD and Cancer Inpatients

\begin{tabular}{|c|c|c|c|c|}
\hline Incidence site of venous thrombosis in IBD and cancer inpatient & IBD $(n=340)$ & UC $(n=89)$ & $C D(n=251)$ & Gl cancer $(n=557)$ \\
\hline Deep venous thrombosis of lower limbs and/or pulmonary artery embolism & $4(16.7)$ & 3 & 1 & $8(57.1)$ \\
\hline Cerebral vein sinus thrombosis & $2(8.3)$ & 2 & 0 & 0 \\
\hline Thrombosis related central venous catheter & $12(50.0)$ & 6 & 6 & $6(42.9)$ \\
\hline Total & 24 & 15 & 9 & 14 \\
\hline
\end{tabular}

Values are presented as number (\%) or number.

$\mathrm{Gl}$ gastrointestinal. 
Table 4. Proportion and Sites of Cases with Symptomatic or Asymptomatic VTE

\begin{tabular}{lcccc}
\hline & Total no. of VTE cases & Symptomatic VTE & Asymptomatic VTE & Proportion of asymptomatic VTE (\%) \\
\hline IBD (L, CVS, PV, CVC) & $24(4,2,6,12)$ & $6(0,2,0,4)$ & $18(4,0,6,8)$ & 75.0 \\
Gl cancer (L, CVS, PV, CVC) & $14(8,0,0,6)$ & $3(1,0,0,2)$ & $11(7,0,0,4)$ & 78.5 \\
Other Gl disease $(L, C V S, P V, C V C)$ & $5(1,0,0,4)$ & $1(1,0,0,0)$ & $4(0,0,0,4)$ & 80.0 \\
\hline
\end{tabular}

$V T E$, venous thromboembolism; $L$, deep venous thromboembolism in lower limbs and/or pulmonary artery embolism; CVS, cerebral vein sinus thrombosis; PV, portal and mesenteric venous thrombosis; $\mathrm{CVC}$, thrombosis related central venous catheter; $\mathrm{Gl}$, gastrointestinal.

vena cava (IVC) filter, catheter extraction, surgery, or no therapy against VTE, according to the condition of IBD or other digestive diseases. The period until the detection of VTE, treatment for VTE, and hemorrhagic complications were collected in VTE group in IBD inpatients. In addition, the in-hospital mortality associated with VTE was compared between the VTE and non-VTE groups.

\section{Statistical Analyses}

Analyses were performed using EZR (Saitama Medical Center, Jichi Medical University, Saitama, Japan), which is a graphical user interface for R (The R Foundation for Statistical Computing, Vienna, Australia). Fisher exact test and a chi-square test were applied to compare the incidence of VTE among groups. An unpaired $t$-test, Mann-Whitney $U$ test, and chi-square test were applied to compare patient characteristics and laboratory data between IBD patients with and without VTE. The cutoff values of the laboratory data with significant differences detected were determined by a receiver operating characteristics (ROC) analysis. A logistic regression analysis was applied to survey independent risk factors of IBD with VTE, which were detected by entering the significant factors revealed in the univariate analysis into a multivariate model. A $P$-value $<0.05$ was considered statistically significant.

\section{RESULTS}

\section{Incidence and Site of VTE}

VTE was observed in $7.1 \%(24 / 340)$ with IBD patients, which was significantly higher than the rates of $2.5 \%$ (14/557) with cancer of the GI tract and $0.88 \%(5 / 569)$ with other GI tract diseases $(P<0.0001$, respectively) (Fig. 2$)$. IBD patients with VTE included 4 patients with deep venous thrombosis (DVT) of the lower limb and/or pulmonary thromboembolism, 2 with cerebral venous sinus thrombosis (CVT), 6 with PVT, and 12 with thrombosis related to a cen-
Table 5. A Univariate Analysis of the Characteristics between IBD Inpatients with and without VTE

\begin{tabular}{|c|c|c|c|}
\hline Characteristics & $\begin{array}{l}\text { VTE group } \\
(n=24)\end{array}$ & $\begin{array}{c}\text { Non-VTE } \\
\text { group }(n=316)\end{array}$ & $P$-value \\
\hline$\overline{\text { Age (yr) }}$ & $49.0 \pm 20.7$ & $38.6 \pm 14.6$ & $0.0180^{\mathrm{a}}$ \\
\hline Age $>50 \mathrm{yr}$ & $11(44.0)$ & $58(18.4)$ & $0.0012^{b}$ \\
\hline Sex (male/female) & $14 / 10$ & $221 / 94$ & $N S^{\mathrm{a}}$ \\
\hline BMI $\left(\mathrm{kg} / \mathrm{m}^{2}\right)$ & $20.0 \pm 3.1$ & $20.1 \pm 3.1$ & $\mathrm{NS}^{\mathrm{a}}$ \\
\hline Disease duration (yr) & $9.5 \pm 10.1$ & $11.7 \pm 9.0$ & $\mathrm{NS}^{\mathrm{a}}$ \\
\hline \multicolumn{4}{|l|}{ Disease type } \\
\hline UC (P/L/T) & $0 / 2 / 13$ & 0/8/66 & $N S^{a}$ \\
\hline $\mathrm{CD}(\mathrm{I} / \mathrm{IC} / \mathrm{C})$ & $3 / 6 / 0$ & $89 / 145 / 8$ & $N S^{\mathrm{a}}$ \\
\hline \multicolumn{4}{|l|}{ Disease activity } \\
\hline UC (CAI) & $9.8 \pm 3.5$ & $8.2 \pm 3.9$ & $N S^{a}$ \\
\hline $\mathrm{CD}$ (CDAI) & $208.1 \pm 110.9$ & $178.8 \pm 78.1$ & $N S^{a}$ \\
\hline Smoking & $5(20.8)$ & $83(26.3)$ & $N S^{b}$ \\
\hline History of thrombosis & 0 & $11(3.5)$ & $\mathrm{NS}^{\mathrm{b}}$ \\
\hline CV catheter & $17(70.8)$ & $87(27.6)$ & $<0.0001^{b}$ \\
\hline Prednisolone & $10(41.6)$ & $30(9.5)$ & $<0.0001^{b}$ \\
\hline Tacrolimus/cyclosporine & $2(8.3)$ & $10(3.2)$ & $N S^{b}$ \\
\hline Thiopurine & $5(20.8)$ & $100(31.7)$ & $N S^{b}$ \\
\hline Anti-TNF- $\alpha$ antibody & $11(45.8)$ & $216(68.6)$ & $N S^{b}$ \\
\hline Plasmacytoapheresis & $3(12.5)$ & $13(4.1)$ & $N S^{b}$ \\
\hline Surgery & $10(41.6)$ & $41(13.0)$ & $<0.0001^{b}$ \\
\hline
\end{tabular}

Values are presented as mean \pm SD or number $(\%)$.

anpaired $t$-test.

${ }^{b}$ Chi-square test.

VTE, venous thromboembolism; $P / L / T$, proctitis/left sided colitis/total colitis type; I/IC/C, ileitis/ileo-colitis/colitis type; CAl, Clinical Activity Index; CV, central venous; TNF, tumor necrosis factor.

tral venous catheter (Table 3). VTE with UC was observed in $16.9 \%$ (15/89), 3 of which had DVT of the lower limb and/or pulmonary thromboembolism, 2 CVT, 4 PVT, 6 thrombosis related to a central venous catheter. VTE with CD was observed in 3.6\% (9/251), 1 of which had DVT of the lower limb and/or pulmonary thromboembolism, 2 PVT, and 6 throm- 
bosis related to a central venous catheter (including 2 patients with home parental nutrition) (Table 3, Fig. 2). Among cases of VTE in GI cancer, VTE in cancer patients with distant metastasis or chemotherapy and in others (including those with early or operable advanced cancer) was observed at rates of $4.0 \%(12 / 303)$ and $0.79 \%(2 / 254)$, respectively. The site of VTE in patients with GI cancer was DVT of the lower limb and/or pulmonary thromboembolism in 8 and thrombosis related to a central venous catheter in 6; no patients developed VTE at the cerebral venous sinus or as PVT. UC patients had a significantly higher risk of VTE than CD patients $(P<0.0001)$. The rate of VTE in UC patients was significantly higher than in those with cancer with distant metastasis or chemotherapy $(4.0 \%)(P<0.0001)$, although the rate in $\mathrm{CD}$ patients was almost equivalent to that in those with cancer with distant metastasis or chemotherapy.

\section{Proportion and Sites of Cases with Symptomatic or Asymptomatic VTE}

Six symptomatic and 18 asymptomatic cases of VTE were detected in IBD inpatients, 3 symptomatic and 11 asymptomatic cases were detected in patients with GI cancer, and 1 symptomatic and 4 asymptomatic cases were detected in patients with other GI diseases (Table 4). The sites of symptomatic and asymptomatic VTE are shown in Tables 5 and 6. Almost all VTE in lower limbs and/or pulmonary artery embolism were asymptomatic in both IBD and GI cancer patients. Both cases of CVT in IBD were symptomatic, while all 6 cases of PVT were asymptomatic.

\section{Risk Factors of VTE}

The patient characteristics and laboratory data were compared between IBD patients with and without VTE. Seven risk factors of VTE with IBD were detected in a univariate analysis. The VTE group with IBD had a higher proportion of older patients $(P=0.0180)$, especially those $>50$ years of age $(P=0.0012)$, $\mathrm{CV}$ catheters $(P<0.0001)$, prednisolone prescription $(P<0.0001)$, and surgery $(P<0.0001)$ (Table 5$)$. On comparing the laboratory data between the 2 groups, the levels of serum albumin were significantly lower $(P=0.0002)$ and those of CRP $(P=0.0260)$ and D-dimer $(P=0.0022)$ significantly higher in the IBD patients with VTE. The cutoff value was serum albumin $\leq 3.0 \mathrm{~g} / \mathrm{dL}(P<0.0001)$, CRP $>1.0$ $\mathrm{mg} / \mathrm{dL}(P=0.015)$, and $\mathrm{D}$-dimer $\geq 1.5 \mathrm{ng} / \mu \mathrm{L}(P=0.0010)$ (Table $6)$, which were determined based on the threshold nearest to the top-left corner of the ROC curve (serum albumin:

Table 6. A Univariate Analysis of Laboratory Data between IBD Inpatients with and without VTE

\begin{tabular}{|c|c|c|c|}
\hline & VTE group $(n=24)$ & Non-VTE group $(n=316)$ & $P$-value \\
\hline White blood cells $(/ \mu \mathrm{L})$ & $6,090(1,850-14,790)$ & $5,030(1,840-21,060)$ & $\mathrm{NS}^{\mathrm{a}}$ \\
\hline Platelet $\left(10^{4} / \mu \mathrm{L}\right)$ & $26.4(9.2-68.8)$ & $28.1(6.3-80.7)$ & $N S^{a}$ \\
\hline Serum albumin (g/dL) & $3.0(2.2-4.0)$ & $3.6(1.6-5.3)$ & $0.0002^{\mathrm{a}}$ \\
\hline Serum albumin $\leq 3.0 \mathrm{~g} / \mathrm{dL}$ & $16(66.6)$ & $68(21.5)$ & $<0.0001^{b}$ \\
\hline $\mathrm{CRP}(\mathrm{mg} / \mathrm{dL})$ & $1.26(0.1-16.9)$ & $0.53(0.1-39.1)$ & $0.0260^{\mathrm{a}}$ \\
\hline $\mathrm{CRP} \geq 1.0 \mathrm{mg} / \mathrm{dL}$ & $16(66.6)$ & $106(33.5)$ & $0.0150^{b}$ \\
\hline BUN (mg/dL) & $10(5-24)$ & $10(2-12)$ & $N S^{a}$ \\
\hline Total cholesterol (mg/dL) & $102(40-259)$ & $85(24-357)$ & $\mathrm{NS}^{\mathrm{a}}$ \\
\hline Triglyceride (mg/dL) & 123 (84-189) & $128(51-258)$ & $\mathrm{NS}^{\mathrm{a}}$ \\
\hline PT-INR & $1.15(0.92-1.54)$ & $1.14(0.85-2.77)$ & $\mathrm{NS}^{\mathrm{a}}$ \\
\hline $\mathrm{aPTT}(\mathrm{s})$ & $32.3(21.7-46.4)$ & $31.0(23.2-52.7)$ & $N S^{a}$ \\
\hline Fibrinogen (mg/dL) & $406.5(183-546)$ & $358(286-827)$ & $\mathrm{NS}^{\mathrm{a}}$ \\
\hline D-dimer (ng/ $\mu \mathrm{L})$ & $2.01(0.09-53.39)$ & $0.81(0.04-13.7)$ & $0.0022^{\mathrm{a}}$ \\
\hline D-dimer $\geq 1.5 \mathrm{ng} / \mu \mathrm{L}$ & $11(45.8)$ & $58(18.3)$ & $0.0010^{b}$ \\
\hline Anti-thrombin III (\%) & $91(63-133)$ & $94(59-139)$ & $N S^{a}$ \\
\hline
\end{tabular}

Values are presented as median (range) or number (\%).

a Mann-Whitney U-test.

${ }^{b}$ Chi-square test.

VTE, venous thromboembolism. 
Table 7. A Multivariate Analysis of the Characteristics and Laboratory Data between IBD Inpatients with and without Venous Thromboembolism

\begin{tabular}{lccc}
\hline \multicolumn{1}{c}{ Characteristics } & OR & 95\% Cl & $\boldsymbol{P}$-value \\
\hline Age $>50 \mathrm{yr}$ & 3.52 & $1.25-9.94$ & 0.018 \\
Central venous catheter & 1.51 & $0.50-4.57$ & 0.462 \\
Prednisolone & 2.41 & $0.72-8.07$ & 0.155 \\
Surgery & 3.19 & $1.02-9.98$ & 0.046 \\
Serum albumin $\leq 3.0 \mathrm{~g} / \mathrm{dL}$ & 2.12 & $0.72-6.28$ & 0.175 \\
CRP $\geq 1.0 \mathrm{mg} / \mathrm{dL}$ & 1.22 & $0.41-3.61$ & 0.718 \\
D-dimer $\geq 1.5 \mathrm{ng} / \mu \mathrm{L}$ & 2.18 & $0.78-6.11$ & 0.136 \\
\hline
\end{tabular}

threshold, $3.10 \mathrm{~g} / \mathrm{dL}$; area under the curve [AUC], 0.6891; CRP: threshold, $1.12 \mathrm{mg} / \mathrm{dL}$; AUC, 0.573; D-dimer: threshold, $1.61 \mathrm{ng} / \mu \mathrm{L}$; AUC 0.66). In a multivariate analysis with a logistic regression analysis, only age $>50$ years $(\mathrm{OR}, 3.52 ; 95 \% \mathrm{CI}$, $1.25-9.94 ; P=0.018)$ and surgery (OR, 3.19; 95\% CI, 1.02-9.98; $P=0.046$ ) were detected as independent risk factors (Table 7). Of 51 cases with IBD who underwent surgeries, 10 VTE cases included 9 elective and 1 emergency surgeries, and 41 non-VTE cases included 38 elective and 3 emergency surgeries. There was no marked difference in the rate of elective and emergent surgeries between IBD with and without VTE.

\section{Clinical Course and Treatment and Hemorrhagic Complications}

The average period until the detection of VTE (from admission) was 18.4 days. Treatment for VTE included anticoagulation therapy in 9 (unfractionated heparin, warfarin, direct oral anticoagulant), fibrinolytic and anticoagulant therapy in 2, IVC filter and anticoagulant therapy in 2, catheter extraction and anticoagulant therapy in 7 , and no therapy (or observation) in 3. No hemorrhagic complications related to antithrombotic therapy occurred. The in-hospital mortality rate associated with VTE in all IBD inpatients was $0.29 \%$ (1/340). The rate in IBD patients with VTE was $4.2 \%(1 / 24)$, and only 1 CVT case resulted in death. In contrast, the rate in IBD patients without VTE was $0 \%$ (Table 8 ).

\section{DISCUSSION}

In this retrospective cohort study, the incidence of VTE in IBD was $7.1 \%$, which was equivalent to the values in previous Western reports ${ }^{1,2,-7}$ and significantly higher than for other digestive disease, including GI cancer, which is known to be associated with a high risk of VTE. In particular, the
Table 8. Clinical Course, Treatment, and Hemorrhagic Complications Associated with VTE in IBD Patients

\begin{tabular}{lc}
\hline \multicolumn{1}{c}{ Variable } & Value \\
\hline Period until detection of VTE (day) & $18.4 \pm 28.8$ \\
In-hospital mortality rate & $4.2(1 / 24)$ \\
VTE group & $0(0 / 316)$ \\
Non-VTE group & \\
Treatment & 3 \\
No therapy/observation & 9 \\
Anticoagulant therapy only & 2 \\
Fibrinolytic/anticoagulant therapy & 2 \\
IVC filter, anticoagulant therapy & 7 \\
Catheter extraction, anticoagulant therapy & 1 \\
Surgery & $0(0 / 24)$ \\
\hline Hemorrhagic complications &
\end{tabular}

Values are presented as mean \pm SD or percent (number/number). VTE, venous thromboembolism; IVC, inferior vena cava.

rate of VTE in UC inpatients was considerably higher than in GI cancer inpatients, which was equivalent to that in CD inpatients. All consecutive inpatients with IBD and GI cancer were surveyed for the presence of VTE using CECT or US, although this study was a retrospective analysis and carried out in difference surveillance programs among patients. Scarpa et al. ${ }^{14}$ reported that the rate of DVT in UC patients was significantly higher than in colorectal cancer patients after colorectal surgery. Our study showed that Japanese IBD inpatients should be paid as much as or more attention concerning VTE than Western IBD patients or cancer patients in real practice.

Sonoda et al. ${ }^{13}$ reported that the incidence of VTE with Japanese IBD patients was $17.0 \%$ in a single-arm prospective study, which was much higher than our results. This discrepancy may be due to those authors performing surveillance of VTE for all patients and using a modality with a high detection sensitivity (magnetic resonance venography, ventilation-perfusion scan). However, in that study, the rate of asymptomatic VTE cases was 75.0\% (6 of 8 VTE cases), which was equal to the results of our present study. Therefore, in Asia including Japan, a prospective study to compare the incidence of VTE in IBD and other digestive disease patients needs to be conducted with the same timing and same modality.

The incidence of VTE was significantly higher with UC than with CD in our study, which was similar to findings in previous studies. ${ }^{9,14,15}$ The activation of the platelet and 
coagulation-fibrinolysis system has been reported to lead to a high frequency of VTE with IBD. ${ }^{16,17}$ Some reports have compared differences in abnormalities in the platelet and coagulation-fibrinolysis system between UC and CD patients; ${ }^{18,19}$ however, the contradictory results failed to explain the difference in the frequency of VTE between UC and CD patients. Nguyen and Sam ${ }^{9}$ predicted that more systemic inflammation caused by colonic disease leads to a more procoagulant state. These findings may explain the higher incidence of VTE in UC compared with CD. An alternate hypothesis is that the higher proportion of prednisolone administration, a common risk factor of VTE, in UC patients (UC 37.1\%, CD 2.7\%) contributed to the greater frequency of VTE in that group.

Thrombosis related to a catheter, PVT and CVT was found to induce an equivalent or greater risk than DVT in the lower limbs and PE in our studies. While a central venous catheter has been predicted to be a common risk factor of $\mathrm{VTE}^{20}$ such catheters are required to be indwelling for the treatment of IBD. We therefore need to be alert for the development of thromboembolism during indwelling of central venous catheters, regardless of the period, as a few patients developed thrombosis around the catheter within a few days in our study (data not shown). PVT related to IBD was reported to be complicated in $1.3 \%$ of cases ${ }^{5}$ and occurred in $4.8 \%$ to $45 \%$ of UC and CD patients post-surgery, ${ }^{21,22}$ with a higher rate of post-total colectomy for UC. ${ }^{22}$ Every IBD patient with any type of bowel resection is thought to have a high risk for PVT and should be given sufficient observation and appropriate screening and preventative measures.

According to Katsanos et al., ${ }^{23}$ CVT was complicated in $1.3 \%$ to $6.4 \%$ in IBD patients, younger than non-IBD patients, and more frequently in UC and active-state patients. It has been reported that headache was experienced in $80 \%$ of CVT patients, and CVT progressed to convulsion, disturbance of consciousness, and neurologic deficit. ${ }^{12}$ The 2 patients with CVT with IBD in our study were both 20-yearolds with UC; 1 case extended to disturbance of consciousness and death as well as headache. CVT should be suspected and investigated quickly when encountering IBD patients with headache. CVT needs to be recognized as thrombosis extending to serious organ failure and death.

Seven and 2 risk factors were detected in univariate and multivariate analyses, respectively, in our study. In addition, UC itself was thought to be a strong risk factor for VTE with IBD because of the markedly high frequency of VTE in those patients compared with CD patients. The risk factors of VTE proposed in previous reports consisted of common risk factors (e.g., obesity, smoking, prolonged immobilization, dehydration, congenital gene mutation of coagulation/fibrinolysis-associated factors), therapeutic procedures related to IBD (abdominal surgeries, central venous catheter, steroid administration), and IBD-specific risk factors (various procoagulant state, types of disease, hospitalization, flare). Older age, central venous catheter, steroid administration, abdominal surgery, ${ }^{2,9}$ and a high level of D-dimer ${ }^{14}$ were similar to previously reported factors; however, a low level of serum albumin and a high level of CRP had not been previously mentioned. A low level of serum albumin or a high level of CRP reflected mainly malnutrition or high disease activity, which needs to be recognized as risk factors of VTE in IBD patients. Among the laboratory data on admission, serum albumin, CRP, and D-dimer were thought to be effective as objective predictive factors of VTE risk. In the present study, the 24 VTE cases in IBD patients consisted of 5 cases of VTE detected on admission and 19 that developed after admission, and D-dimer levels were higher in the patients that developed VTE after admission (data not shown). Therefore, the trend toward a higher D-dimer level on admission in IBD patients with VTE may suggest either existing VTE at admission or the development of VTE after admission. In addition, continuous monitoring of D-dimers was suggested to be useful for the early detection of VTE because of further elevation in the D-dimer levels in IBD inpatients with VTE in this study.

The in-hospital mortality rate was $4.2 \%$ in IBD inpatients with VTE in our study but $0 \%$ in IBD patients without VTE. Nguyen and Sam ${ }^{9}$ previously reported that the in-hospital mortality rate was 37.4 per 1,000 hospitalizations in UC with VTE and 17.0 per 1,000 hospitalizations in CD with VTE, which was considerably higher than in a non-VTE American IBD cohort. An increased risk of in-hospital mortality should be considered in IBD inpatients with VTE.

Several limitations associated with the present study warrant mention. First, this study was a retrospective and singlecenter study. Because of the existence of referral hospitals, there may have been more severe patients with IBD recognized as having a higher risk of VTE. Second, the characteristics among 3 groups who were surveyed in our study were not matched. One reason for this limitation was that the onset of most IBD patients was earlier than that in patients with cancers and other GI diseases. A higher age is known to be a risk of VTE, so the VTE risk of IBD might be emphasized when age is matched among the groups. Third, the timing and area of surveillance of VTE varied among patients. VTE defined in our study included both symptomatic and asymp- 
tomatic thrombosis. Asymptomatic thrombosis consisted of that detected at screening for VTE and that detected incidentally at CECT for purposes other than diagnosing VTE. The present study revealed that $75 \%$ of all VTE were asymptomatic in IBD inpatients as well as patients with cancer and other benign diseases. However, it is difficult to determine the actual incidence of asymptomatic VTE in IBD inpatients retrospectively due to insufficient screening procedures, as mentioned above. To resolve these limitations and clarify the frequency and risk factors of VTE in IBD patients, multicenter prospective studies need to be conducted in Asia in the future.

In conclusion, we found the frequency of VTE in IBD to be high, especially in UC, and identified the associated risk factors. The findings in the present study will help clarify the actual state of VTE with IBD in Japanese and Asian IBD populations. Future prospective studies to clarify the incidence and risk factors of VTE with IBD will aid in the development of prophylaxis for groups at high risk of developing VTE.

\section{FINANCIAL SUPPORT}

This paper was supported by Intractable Disease Health and Labour Sciences Research Grants from the Ministry of Health, Labour and Welfare.

\section{CONFLICT OF INTEREST}

No potential conflict of interest relevant to this article was reported.

\section{AUTHOR CONTRIBUTION}

K.A. and M.F. provided major input into the conceptual development of the studies, wrote the manuscript and supervised all of the investigations. Y.N., Y.I., Y.S., T.I., M.I., Keitaro Takahashi, Kazuyuki Tanaka, A.S., N.U. and S.K managed and treated the enrolled patients and collected and analyzed the data, K.M., Y.M. and T.O. helped to design the studies, interpret the data, and prepare/review the manuscript. All of the authors read and approved the final manuscript.

\section{REFERENCES}

1. Papa A, Papa V, Marzo M, et al. Prevention and treatment of venous thromboembolism in patients with IBD: a trail still climbing. Inflamm Bowel Dis 2015;21:1204-1213.
2. Purnak T, Yuksel O. Overview of venous thrombosis in inflammatory bowel disease. Inflamm Bowel Dis 2015;21:1195-1203.

3. Danese S, Papa A, Saibeni S, Repici A, Malesci A, Vecchi M. Inflammation and coagulation in inflammatory bowel disease: the clot thickens. Am J Gastroenterol 2007;102:174-186.

4. Zitomersky NL, Verhave M, Trenor CC 3rd. Thrombosis and inflammatory bowel disease: a call for improved awareness and prevention. Inflamm Bowel Dis 2011;17:458-470.

5. Talbot RW, Heppell J, Dozois RR, Beart RW Jr. Vascular complications of inflammatory bowel disease. Mayo Clin Proc 1986;61:140-145.

6. Webberley MJ, Hart MT, Melikian V. Thromboembolism in inflammatory bowel disease: role of platelets. Gut 1993;34:247251.

7. Sloan WP Jr, Bargen JA, Gage RB. Life histories of patients with chronic ulcerative colitis: a review of 2,000 cases. Gastroenterology 1968;54:Suppl:819-822.

8. Miehsler W, Reinisch W, Valic E, et al. Is inflammatory bowel disease an independent and disease specific risk factor for thromboembolism? Gut 2004;53:542-548.

9. Nguyen GC, Sam J. Rising prevalence of venous thromboembolism and its impact on mortality among hospitalized inflammatory bowel disease patients. Am J Gastroenterol 2008;103:22722280.

10. Grainge MJ, West J, Card TR. Venous thromboembolism during active disease and remission in inflammatory bowel disease: a cohort study. Lancet 2010;375:657-663.

11. Sinagra E, Aragona E, Romano C, et al. The role of portal vein thrombosis in the clinical course of inflammatory bowel diseases: report on three cases and review of the literature. Gastroenterol Res Pract 2012;2012:916428. doi: 10.1155/2012/916428.

12. Cognat E, Crassard I, Denier C, Vahedi K, Bousser MG. Cerebral venous thrombosis in inflammatory bowel diseases: eight cases and literature review. Int J Stroke 2011;6:487-492.

13. Sonoda K, Ikeda S, Mizuta Y, Miyahara Y, Kohno S. Evaluation of venous thromboembolism and coagulation-fibrinolysis markers in Japanese patients with inflammatory bowel disease. J Gastroenterol 2004;39:948-954.

14. Scarpa M, Pilon F, Pengo V, et al. Deep venous thrombosis after surgery for inflammatory bowel disease: is standard dose low molecular weight heparin prophylaxis enough? World J Surg 2010;34:1629-1636.

15. Alatri A, Schoepfer A, Fournier N, et al. Prevalence and risk factors for venous thromboembolic complications in the Swiss Inflammatory Bowel Disease Cohort. Scand J Gastroenterol 2016;51:1200-1205. 
16. Stadnicki A. Involvement of coagulation and hemostasis in inflammatory bowel diseases. Curr Vasc Pharmacol 2012;10:659669.

17. Chiarantini E, Valanzano R, Liotta AA, et al. Hemostatic abnormalities in inflammatory bowel disease. Thromb Res 1996;82:137-146.

18. Kjeldsen J, Lassen JF, Brandslund I, Schaffalitzky de Muckadell OB. Markers of coagulation and fibrinolysis as measures of disease activity in inflammatory bowel disease. Scand J Gastroenterol 1998;33:637-643.

19. Weber P, Husemann S, Vielhaber H, Zimmer KP, Nowak-Göttl U. Coagulation and fibrinolysis in children, adolescents, and young adults with inflammatory bowel disease. J Pediatr Gastroenterol Nutr 1999;28:418-422.
20. Heit JA. The epidemiology of venous thromboembolism in the community: implications for prevention and management. J Thromb Thrombolysis 2006;21:23-29.

21. Fichera A, Cicchiello LA, Mendelson DS, Greenstein AJ, Heimann TM. Superior mesenteric vein thrombosis after colectomy for inflammatory bowel disease: a not uncommon cause of postoperative acute abdominal pain. Dis Colon Rectum 2003;46:643-648.

22. Baker ME, Remzi F, Einstein D, et al. CT depiction of portal vein thrombi after creation of ileal pouch-anal anastomosis. Radiology 2003;227:73-79.

23. Katsanos AH, Katsanos KH, Kosmidou M, Giannopoulos S, Kyritsis AP, Tsianos EV. Cerebral sinus venous thrombosis in inflammatory bowel diseases. QJM 2013;106:401-413. 